\title{
A Study on the Influence of the Forming Strategy on the Main Strains, Thickness Reduction, and Forces in a Single Point Incremental Forming Process
}

\author{
Adrian Blaga and Valentin Oleksik \\ Lucian Blaga University of Sibiu, Faculty of Engineering, Victoriei Boulevard 10, 550024 Sibiu, Romania \\ Correspondence should be addressed to Valentin Oleksik; valentin.oleksik@ulbsibiu.ro
}

Received 25 May 2013; Revised 17 August 2013; Accepted 18 August 2013

Academic Editor: Tao Zhang

Copyright (C) 2013 A. Blaga and V. Oleksik. This is an open access article distributed under the Creative Commons Attribution License, which permits unrestricted use, distribution, and reproduction in any medium, provided the original work is properly cited.

\begin{abstract}
This paper comprises an experimental study for a complex geometry part obtained by incremental forming. Due to the process complexity (the presence of forces on three directions-a vertical one and two in the blank's plane), a three axes CNC milling machine, capable of describing the complex paths covered by the punch for obtaining the truncated cone-shaped parts, has been chosen. To obtain a truncated cone, three different trajectories were selected: in first and second variants after each vertical press having a constant step, the punch covers a circular path. The differences show that the following circular trajectory can start at the same point or can be shifted at an angle of $90^{\circ}$ from the previous press point. In the last variant, the punch performs a spatial spiral trajectory. The main objective of our study was to determine the optimal forming strategy, by shifting the press position of the punch and the path it follows to obtain a truncated cone through single point incremental forming. Thus, the strain distribution can be homogeneous, and the thickness reduction and the process forces are minimal.
\end{abstract}

\section{Introduction}

The incremental sheet metal forming process is a modern method of material forming processes, with huge potential regarding the degree of flexibility and customization of the parts made through this process. Within the analysed process, the strain is created by means of a numerically controlled punch. The punch follows a trajectory defined by the CNC program of a milling machine on which strains are produced. The material is strained locally only in the area that comes into contact with the punch, hence the name incremental forming, the material being formed gradually.

With regard to single point incremental forming, Pohlak et al. [1] developed a formula to calculate forces by taking into account the anisotropy of the material as well (which was described using Hill's higher order mathematical model).

Another approach to the single point incremental forming process has been identified in Silva's analytical studies [2], which, in order to explain the phenomenon of crack occurring in a component part, consider the behaviour of the material in incremental forming to be similar to that of a membrane.

Multistage deformation path strategies for single point incremental forming (SPIF) are revised with the purpose of improving sheet thickness distribution and forming a vertical wall surface for cylindrical cups by Liu et al. [3]. In this paper, three basic multistage deformation path strategies have been proposed: incremental part diameter, incremental draw angle, and incremental part height and draw angle. Approximate plane-strain analysis models have been given to provide formability predictions between single-stage and multistage strategies.

Yamashita et al. [4] performed the single point incremental forming numerical simulation (SPIF) to analyse the influence of path type on the forces and accuracy of component parts.

Li et al. [5] present a study based on a finite element method model which was experimentally verified; here, different forming strategies were adopted to form a frustum of cone with a wall angle of $30^{\circ}$ to research the influence 
of the number of forming stages and the incremental wall angle between the two adjacent stages on the formability of incremental forming process. The simulation results included the thickness distribution, the equivalent plastic strain, and the magnitude of springback. The study revealed that with the growth of the number of forming stages, the minimum thickness increases largely, and more uniform thickness distribution is achieved, but springback becomes larger in contrast with a single-pass process.

A deformation analysis of incremental forming process is presented by Cui et al. [6]. The analysis presented here includes the development of an analytical model for strain distributions based on part geometry and tool paths, numerical simulations of the forming process with LS-DYNA, and experimental validation of strain predictions using digital image correlation (DIC) techniques. Three kinds of parts hyperbolic cone, skew cone, and elliptical cone, are constructed and used as examples for the study. Analytical, numerical, and experimental results are compared, and good correlations are found.

Bambach et al. [7] analysed the forming of a cone-shaped part using both the explicit and implicit methods with the help of the Abaqus/Standard and Abaqus/Explicit programs.

Silva and Martins [8] propose a combined theoretical and experimental study of two-point incremental forming (TPIF) with partial die. The theoretical developments include an innovative extension of the analytical model for rotational symmetric single point incremental forming, and the experimental work comprised the mechanical characterization of the material and the determination of its formability limits at necking and fracture by means of circle grid analysis.

In the single point incremental forming study, experimental research methods were used to highlight the mechanics of the forming process, the forces required in the forming process, the accuracy of the surface obtained, the formability of various materials, the optimum path of the punch, and so forth.

The mechanics of the incremental forming process was examined by comparing single point and two points incremental forming from Jackson's experimental perspective [9].

Experimental research on single point incremental forming was mainly directed at determining the forces involved in the process. Minutolo et al. [10] performed a comparative numerical simulation experimental study on the variation of forces involved in single point incremental forming. Duflou et al. [11] analysed the forces involved in single point forming and the influence exerted on these forces by the punch step, punch diameter, material thickness, and wall angle of the workpiece. Fiorentino et al. [12] determined the forces along three directions applied in two points incremental forming with an active element of positive support. Durante et al. [13] studied the influence of the spin velocity of the punch on the forces involved in the process and on the surface roughness of the formed parts. Ambrogio et al. [14] emphasised the importance of controlling the incremental forming process by monitoring the forces involved in this process.

The accuracy of the parts obtained through single point incremental forming was studied by Ambrogio et al. [15], who analysed the influence of process parameters. Various forming strategies for both single point and two points incremental forming, meant to increase the geometrical accuracy of the parts, are also presented by Bambach et al. [16].

Hussain and Gao [17] studied the formability of various materials in single point and two points incremental forming and determined the forming limit diagram typical of these procedures.

Attanasio et al. [18] studied the optimisation of the path followed by the punch in two points incremental forming in terms of dimensional accuracy, surface quality, and piece thickness. Two types of paths were considered: a constant step path along a vertical direction and another one with a constant scallop height. Punch path optimisation in single point incremental forming is studied by Rauch et al. [19]. In the first stage, he shows the impact that the punch path type and other parameters have on the accuracy of the parts obtained through incremental forming. Based on this analysis, he developed an intelligent CAM system that optimises the punch path by taking into account the process constraints. The optimisation follows two strategies: one based on previously determined forces and the other based on previous measurements of piece accuracy.

Fu et al. [20] present an iterative algorithm of closedloop control for correcting tool path of the single point incremental forming, based on fast Fourier transform. The springback, after unloading, of the sheet metal parts formed with the trial and corrected tool paths is obtained with finite element model (FEM) simulation.

This paper aims at studying the influence of the forming strategy (by choosing the area of punch press in the first step and also its subsequent path) on the main strains, thickness reduction, and forces to obtain truncated cone-shaped parts.

\section{Materials and Methods}

Experimental investigations were carried out on a three axes CNC milling machine, DMG Veco, capable of describing the complex paths covered by the punch in order to obtain truncated cone-shaped parts. The milling machine table was fixed with an elastic element enabling the measurement of forces, and the elastic element was fitted with the die. The punch was fitted on the crunch of the CNC milling machine.

The experimental layout is presented in Figure 1. The data acquisition system allows the simultaneous recording of forming force values for two components, a vertical one and a horizontal one $\left(F_{z}\right.$ and $\left.F_{x}\right)$, at a capture frequency of $100 \mathrm{~Hz}$. The capture time varied depending on the type of path. For measurement only two components, one vertical and one in sheet metal plane, have been chosen due to the symmetry of truncated cone parts.

The second horizontal component has the same variation as the first but out of phase with an angle of 90 degrees, for which it was not taken into account in this study. The signal acquired from the analogue digital board KPCI 3108 can be processed, filtered, and saved via a virtual instrument created using the Matlab program. The actual forming force values of the components were calculated by means of the 


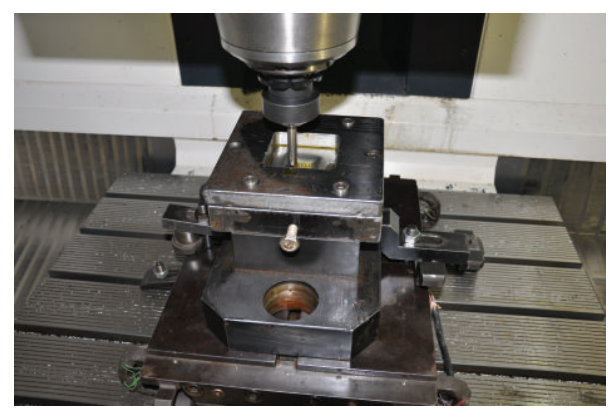

Figure 1: The experimental layout.

interdependence ratio between the electrical signals received and force magnitude, determined using calibration curves.

The die used in the incremental forming consists of a support plate, a port die plate, a die, and a blankholder. The die is circular, with a diameter of $D_{p l}=55.5 \mathrm{~mm}$ and a radius of $R_{p l}=6 \mathrm{~mm}$. The hemispherical punch used to produce the pieces has a diameter $D_{p}=8 \mathrm{~mm}$.

To measure the strains "offline," an Argus optical measurement system produced by Gom was used (Figure 2). The samples were electrochemically marked before forming. To mark them, a network of circles with a diameter of $1 \mathrm{~mm}$ and $2 \mathrm{~mm}$ distance between them was selected. The samples were marked on the opposite side of the area that came into contact with the punch.

The strains were measured with a camera having a lens's focal length of $12 \mathrm{~mm}$. The pieces were placed on a turntable. Four coded bars and 12 markers enabling the calibration of the optical system were stacked on the pieces. Strain accuracy for the Argus optical measurement system is $0.1 \%$. The program installed on the optical analyser can measure the strains in three ways: the engineering strains, the logarithmic strains, and the Green strains. In this paper, we determined the logarithmic strains.

\section{Results and Discussion}

3.1. Strain Distribution and Thickness Reduction. The main objective of the present study was to determine the optimal forming strategy by shifting the penetrating position of the punch and the path it follows to obtain a truncated cone through single point incremental forming, so that the strain distribution can be homogeneous and thickness reduction minimal. To carry out the tests, a DC04 steel sheet (SR EN 10130-2000) with a thickness of $0.7 \mathrm{~mm}$ was used. DC04 is commercial deep-drawing steel commonly used in automotive industry. The material elasticity modulus is $E=7.10 e+$ $5 \mathrm{MPa}$, and the transversal contraction coefficient is $v=0.34$, while the yield stress is $\sigma_{y}=165 \mathrm{MPa}$, the strength coefficient $K=531 \mathrm{MPa}$, and the hardening coefficient $n=0.2397$. The anisotropic characteristic width to thickness strain ratio values are $r_{00}=1.77 ; r_{45}=1.43 ; r_{90}=2.14$. Determination of material constants was made based on uniaxial tensile tests. The tensile tests were performed on an Instron 5587 testing machine, provided with a computer interfacing with the load frame, capable of keeping a constant rate and changing load

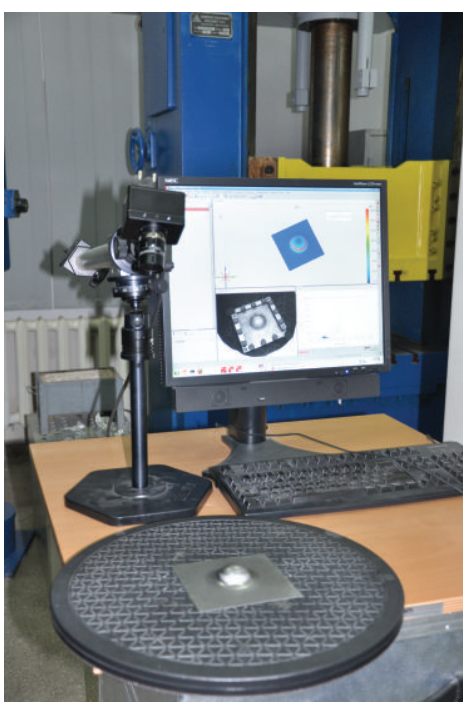

Figure 2: The Argus optical measurement system used for strain and thickness reduction evaluation.

cells allowing accuracy to be within + or $-0.5 \%$ of reading down to $1 / 500$ of load cell capacity. In order to determine the anisotropic characteristics, during the uniaxial tension tests, an online optical extensometer Aramis was used.

Because the Argus measurement system allows precisely the determination of the main strains and thickness reduction on a calibrated area, limited from dimensional point of view, it was chosen for this study to achieve some small pieces, so that the accuracy of the results is as high as possible. The diameter of the large base of the truncated cone was chosen such that $d=44 \mathrm{~mm}$. So, the truncated cone formed has a maximum height of $12 \mathrm{~mm}$ for a wall angle of $55^{\circ}$. A low height part has been chosen also because high parts are hard to measure in a single clamping, requiring many clamping and combining measurements in the program that would have led to imprecise results. To obtain this shape, three different trajectories as shown in Figure 3 were chosen. The first two variants (Figures 3(a) and 3(b)) differ in terms of the successive positions of punch presses into the material. After each vertical press with a constant step of $1 \mathrm{~mm}$, the punch covers a circular path. The following forming circular trajectory can start in the same spot (variant V1) (Figure 3(a)) or can be shifted at an angle of $90^{\circ}$ from the previous press point (variant V2) (Figure 3(b)) depending on the used trajectory. If the last variant (V3) is used, the punch performs a spatial spiral motion, as shown in Figure 3(c). The step between two successive paths in this case, too, measured $1 \mathrm{~mm}$. Practically, the punch performs continuous press on the material in all three directions.

The experimental researches were repeated five times for each type of the chosen trajectories both in terms of the determination of main strains and thickness reduction as well as forces in two directions so that any errors will be eliminated.

Table 1 presents the maximum values of the main strains and thickness reduction obtained from the measurements of 


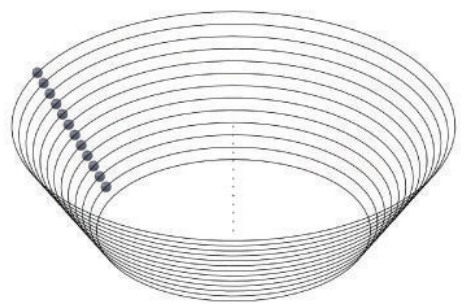

(a)

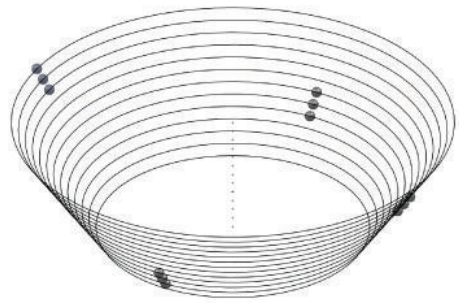

(b)

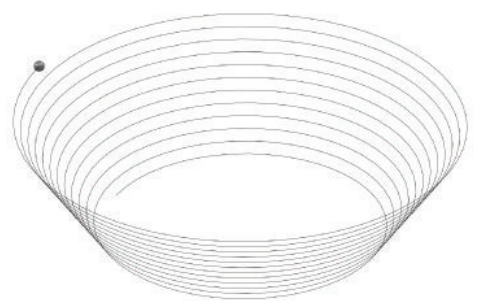

(c)

FIgURE 3: The three different trajectories used for the produced parts (a) V1, (b) V2, and (c) V3.

TABLE 1

\begin{tabular}{lccc}
\hline Variant & $\begin{array}{c}\varepsilon_{1 \max } \\
{[\mathrm{mm} / \mathrm{mm}]}\end{array}$ & $\begin{array}{c}\varepsilon_{2} \max \\
{[\mathrm{mm} / \mathrm{mm}]}\end{array}$ & $\begin{array}{c}\text { Thickness reduction } \\
{[\mathrm{mm} / \mathrm{mm}]}\end{array}$ \\
\hline V1 & 0,5928 & 0,163 & 0,679 \\
V2 & 0,6119 & 0,1129 & 0,673 \\
V3 & 0,502 & 0,113 & 0,5986 \\
\hline
\end{tabular}

the three path types. In the tables and figures, the results of experimental determinations with the values closest to mean value of the five experiments are presented (the median values). Median values were presented in order to reach a good correlation between the results presented in the tables and figures.

It can be observed that, for all three path types, the major strain (Figure 4) and thickness reduction (Figure 6) have maximum values in a plane that is parallel to $x \mathrm{O} y$. This plane corresponds to a circular path deflected at about $9.5 \mathrm{~mm}$ from the truncated cone base.

For both the path with successive presses in the same area (V1) and the one with successive presses shifted at an angle of $90^{\circ}$ from the previous press (V2), we notice that the value of the major strain does not have a homogenous variation on the circular path (Figures 4(a) and 4(b)). In variant V1, the strain reaches a maximum value at the press point of the punch into the material and has continuous decreasing variation along the circular path. In variant V2, the major strain reaches maximum local values at the presses points, and these peak values also influence the strains occurring during the next forming path. As a result, the major strain on a path is no longer continuous but fluctuates.

Unlike the other two variants considered, in a spiral trajectory (V3), the major strain values are relatively constant or decrease much more gently along the same circular path. The major strain maximum value is 0.6199 for variant (V2) and 0.5928 for variant V1. The lowest of the maximum values reached is found in the spiral trajectory (V3), the major strain being 0.5020 .

The minor strain also reaches a maximum value in the initial point of punch press along a vertical direction for V1 and for the spiral trajectory, while for variant V2 there are four areas of maximum local values corresponding to the press points (Figures $5(\mathrm{a}), 5(\mathrm{~b})$, and $5(\mathrm{c})$ ). If, in the spiral trajectory (V3), minor strain is relatively constant along a section parallel to the truncated cone base, for the other two
TABLE 2

\begin{tabular}{lcc}
\hline Variant & $F_{x \max }[\mathrm{N}]$ & $F_{z \max }[\mathrm{N}]$ \\
\hline V1 & 414 & 1004 \\
V2 & 361 & 928 \\
V3 & 391 & 811 \\
\hline
\end{tabular}

types of paths (V1 and V2), at the end of each circular path, minor strain decreases sharply, resulting in negative values, in the proximity of the press area.

This is due to the material fold that is formed before the punch press in these two types of paths. In the spiral path (V3), the process is continuous, with no repeated punch presses along the vertical and circular directions, leading to the smoothing of the material. The material folding in the area before the punch press is a major disadvantage of the two types of paths (V1 and V2), resulting in reduced accuracy of the formed parts and increased forces involved in the process. The minor strain maximum values are 0.163 for V1, 0.1129 for $\mathrm{V} 2$, and 0.113 for V3.

The thickness reduction has a variation that is similar to the major strain for all three types of paths. The peak value is reached at the points of initial punch press on a vertical direction. Higher values similar to those of the major strain are reached and decrease as the diameter of the circular path reduces (Figure 6). Maximum thickness reduction values are 0.679 for V1, 0.673 for V2, and 0.5986 for a spiral trajectory (V3).

The best variant for manufacturing truncated coneshaped parts in terms of the distribution of strains is the one involving the use of a spiral path, as the distribution of major strains and relative thickness is more homogeneous, and relative thickness has lower values.

3.2. Variation of Forces in Forming a Truncated Cone. Table 2 shows the results obtained by measuring forces along both directions of the coordinate system $O_{x}$ and $O_{z}$.

As regard force values on the horizontal direction $\left(F_{x}\right)$, it can be observed that the maximum value of $414 \mathrm{~N}$ is reached in the case of successive punch presses in the same area (V1). In spiral trajectory (V3), the maximum force value on the $x$ direction is $391 \mathrm{~N}$. The lowest value of the maximum values reached is found in the case of successive presses offset at an angle of $90^{\circ}$ from the previous press (V2), its value being 


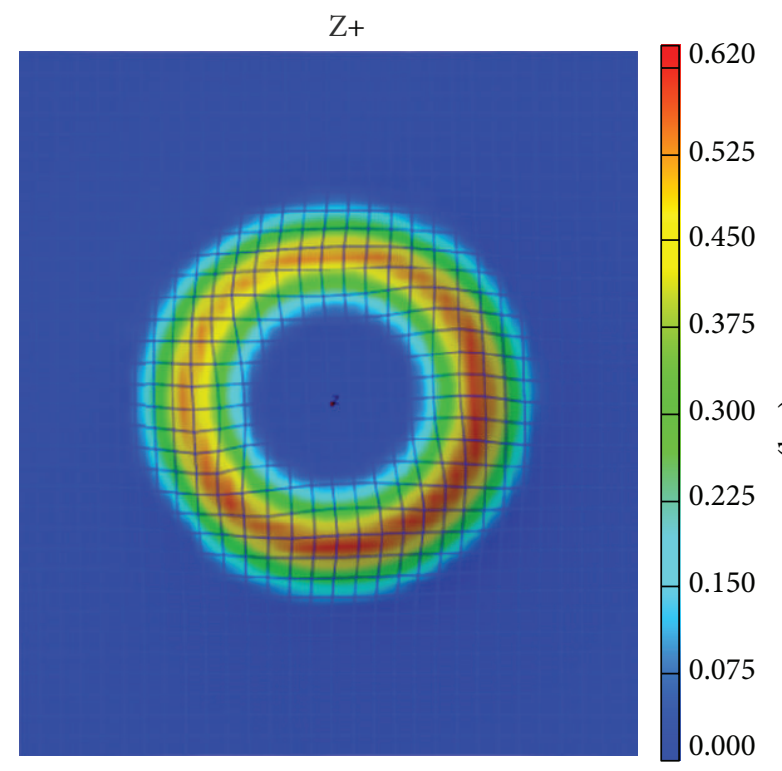

(a)

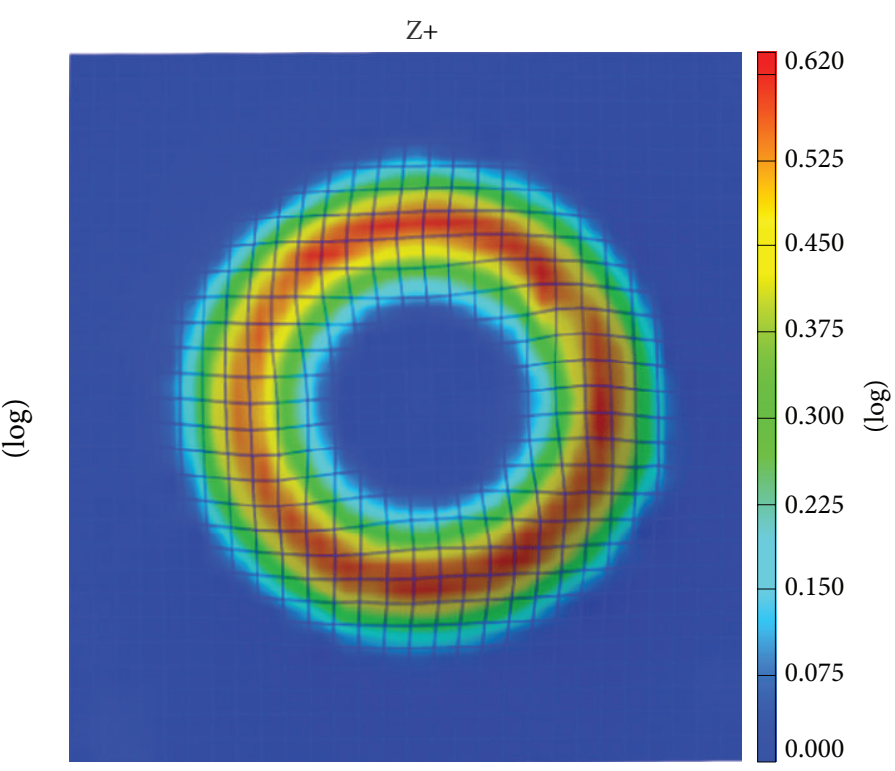

(b)

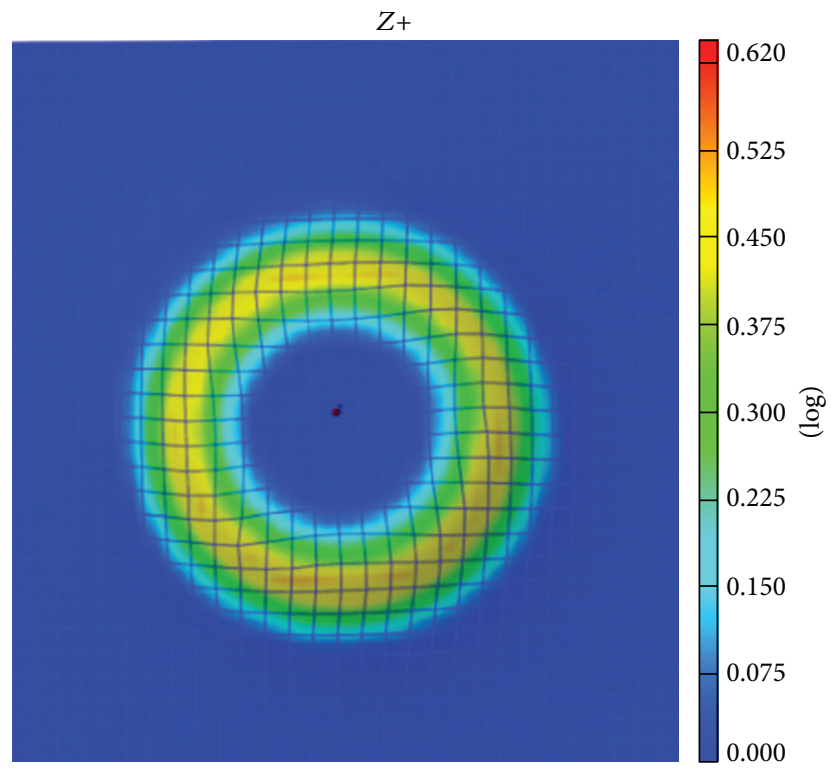

(c)

FIGURE 4: The major strain variation for the specified three different trajectories.

361 N. Figure 7 shows the graphs obtained for the horizontal component of the force $\left(F_{x}\right)$ for all three types of paths.

In the case of successive punch presses in the same area (V1) and the spiral path (V3), the variation of force $F_{x}$ is harmonic with the variable amplitude (Figures $7(\mathrm{a})$ and $7(\mathrm{c})$ ). In both $\mathrm{V} 1$ and V3, the amplitude increase is approximately linear until the maximum is reached, which is located on the last step in variant V3 and on the penultimate step in variant V1. In successive presses offset at an angle of $90^{\circ}$ from the previous press (V2), the force variation along the horizontal direction $F_{x}$ is uneven; due to the particularity of the path, with local peaks being reached somewhere near midpath and then remaining relatively constant (Figure 7(b)).
As to the variation of component $F_{x}$ in the first two types of paths V1 and V2, we can state that each punch press reaches a local peak, and at the end of each circular path, there is a sharp drop in this peak value (Figure 7(b)). This abrupt drop in variant V2 almost disappears after covering half of the path; with the force variation continuing to be similar to variant V3, which has a smooth trend, without sharp increases or decreases in force values. This favourable impact-free trend is less demanding for the feed box of the milling machine.

Figure 8 shows graphs drawn for the vertical component of the force $\left(F_{z}\right)$ for all three types of paths. Regarding the force values along the vertical direction $\left(F_{z}\right)$, the maximum value of $1004 \mathrm{~N}$ is reached in the case of the variant with 


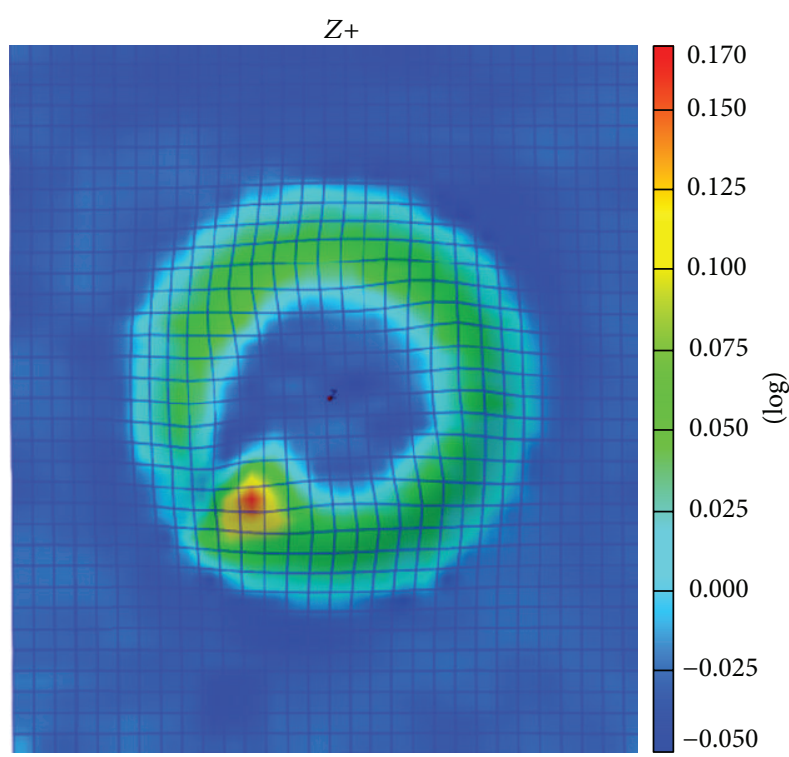

(a)

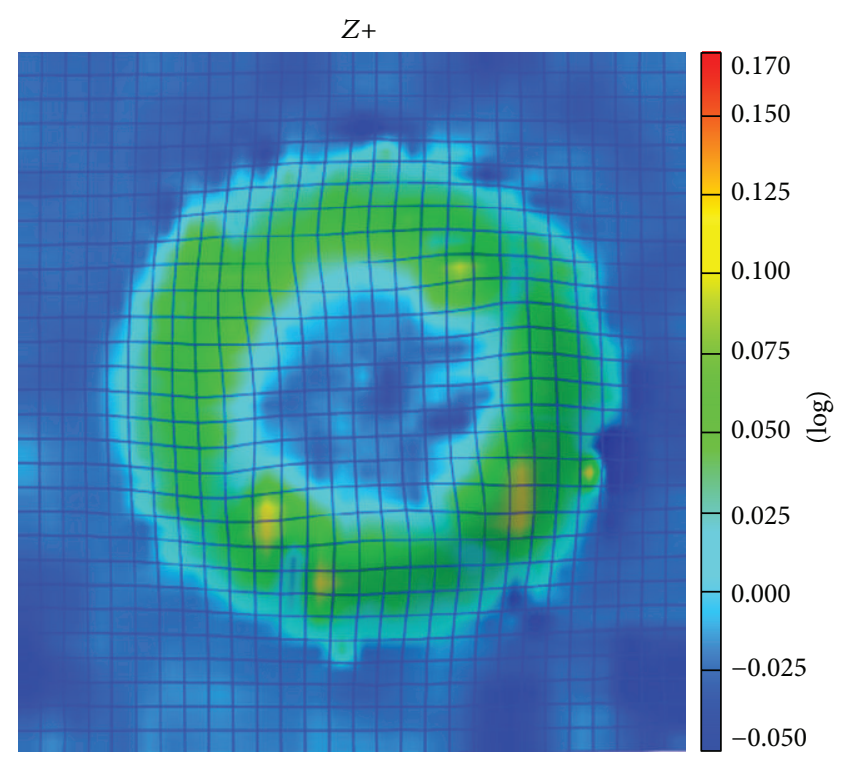

(b)

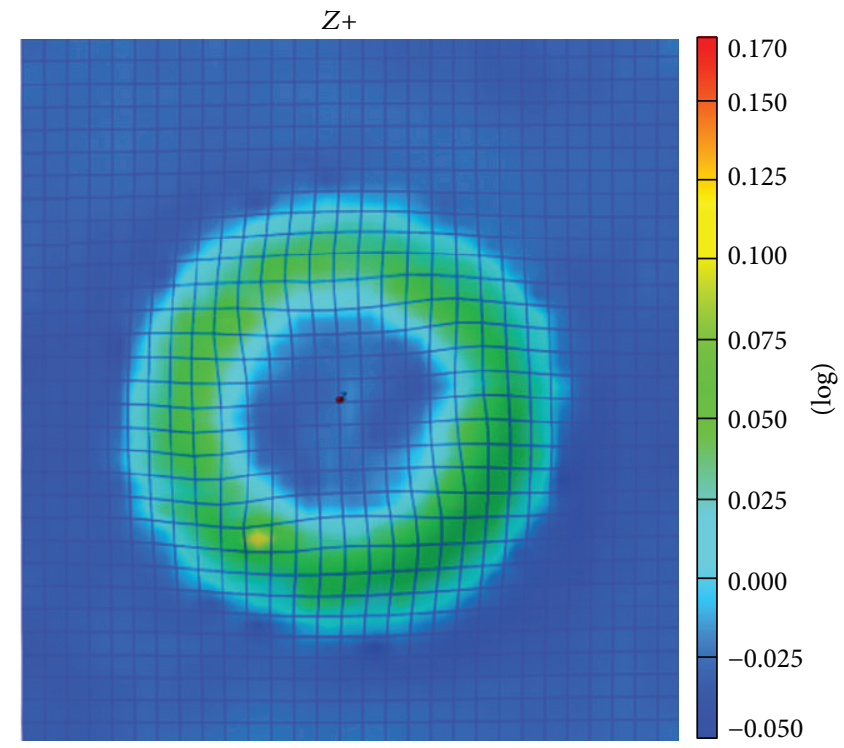

(c)

Figure 5: The minor strain variation for the specified three different trajectories.

successive presses in the same area (V1). The variant with successive presses offset at an angle of $90^{\circ}$ from the previous entry (V2) attains a maximum force value on the $\mathrm{O}_{z}$ direction of $928 \mathrm{~N}$. The lowest value of the maximums reached is found in V3, its value being $811 \mathrm{~N}$. It can be clearly interpreted that the strain variant after a spiral path leads to forces whose maximum value is significantly reduced compared to the other two types of paths.

In the variant with successive presses in the same area (V1), vertical force variation reaches a local maximum after each vertical punch press, followed by a sudden drop and then by a smooth decrease until a circular path is completed, when there is another local minimum. This variation is repeated at each work cycle until the peak force value along the vertical direction is reached. This peak is reached near the area where the last vertical steps are performed. Here, it is noticed that the differences between the local highs and lows of each circular path stay approximately constant throughout the entire path.

In the variant with successive presses offset at an angle of $90^{\circ}$ from the previous entry (V2), the variation of the vertical component of the force is similar to that of variant V1 for the areas where full circles are completed. The only difference is the emergence of areas with minimum values where the punch performs a repositioning movement of the press point.

In the variant where the geometry of the part is described by a spiral path (V3), the variation of the vertical component of the force is smooth with no local maximum or minimum. 


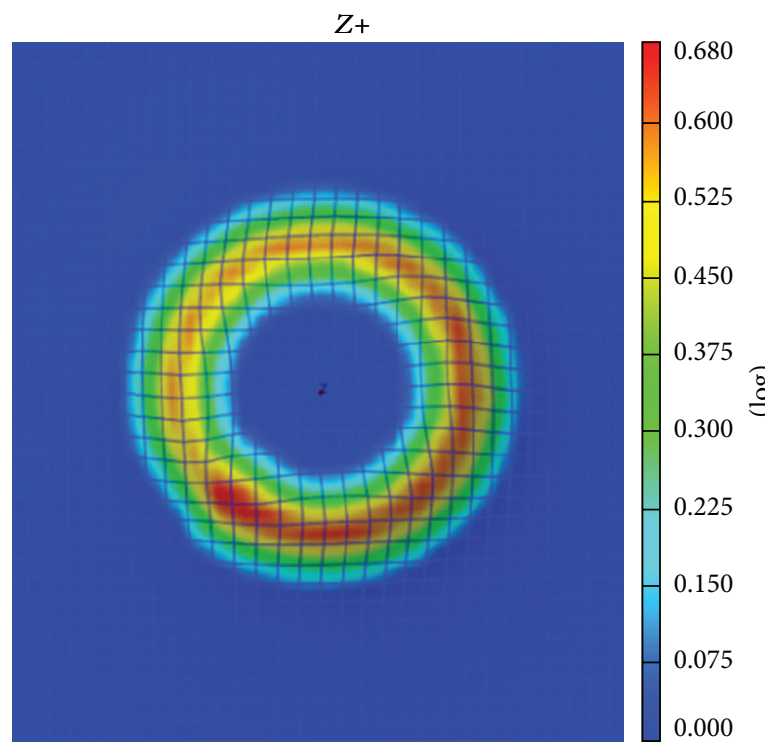

(a)

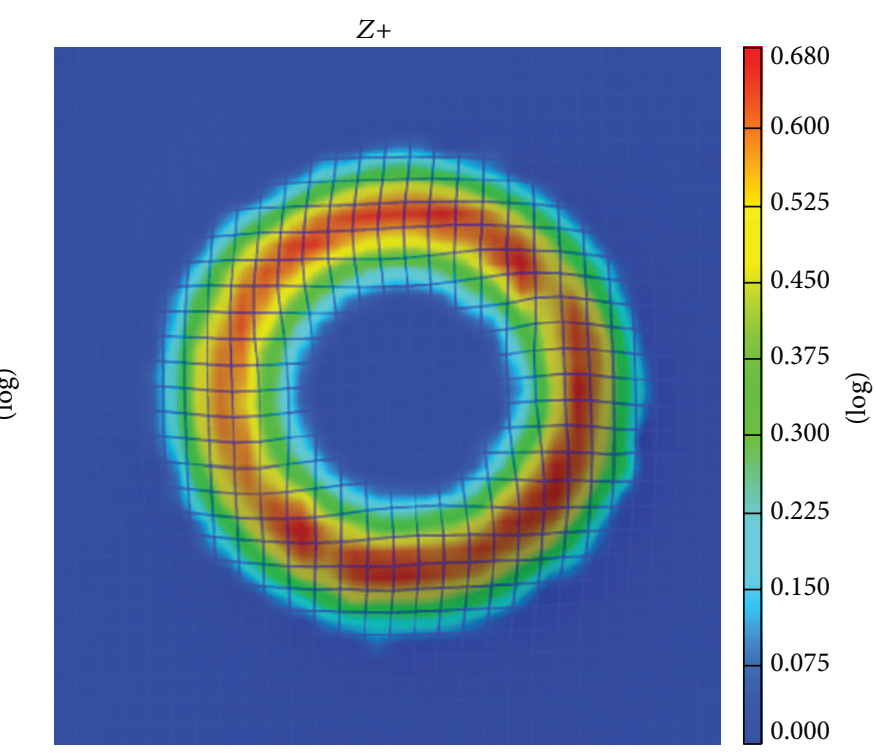

(b)

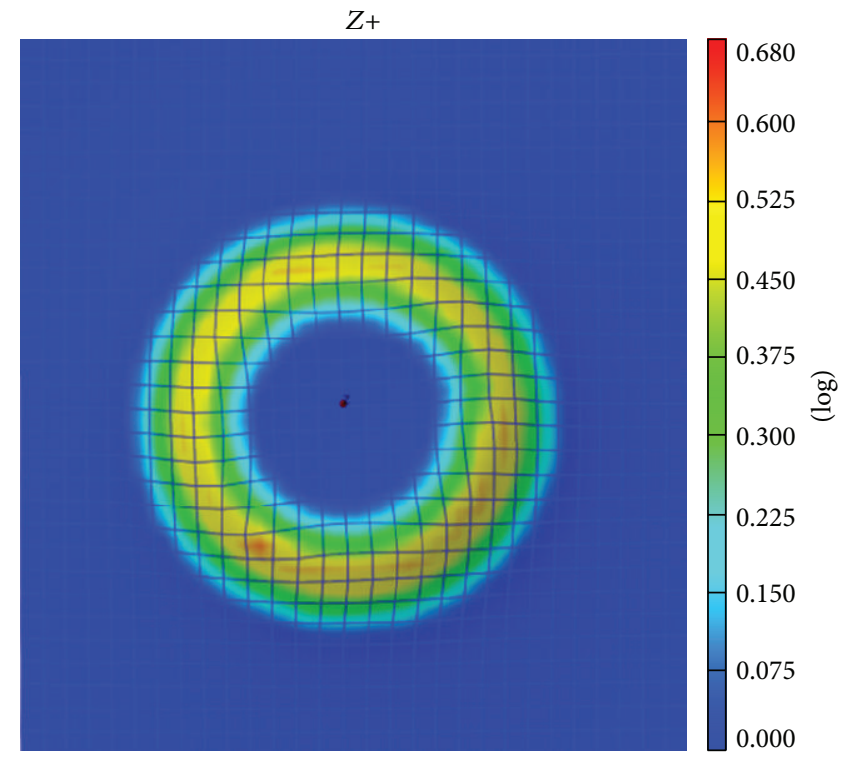

(c)

FIGURE 6: The thickness reduction variation for the specified three different trajectories.

The vertical component of the force increases until reaching a maximum value (close to the area where the last steps are performed) and then remains relatively constant until just before the end of part processing. As in the case of the horizontal component, it is noticed that the best processing variant is the spiral path, which has smaller peaks and a smooth variation.

3.3. Precision of Parts in Forming a Truncated Cone. Although SPIF is a very flexible process and requires very simple equipment, it has a major disadvantage, namely, precision of the obtained parts. To determine which of the three paths is better in terms of accuracy of the resulting piece, the same optical measuring system Argus was used. With this device, 3D geometries of measured parts were performed after deformation. These geometries were exported in STL format and compared to the model that was supposed to be achieved. To highlight which of the three paths is better to obtain a more accurate part, the pieces were sectioned through the area with the largest deviations from the profile (Figure 9(a)). As can be seen from the figure, for all paths a sheet convexity close to the major base of the part is presented (Figure 9(b)).

From this point of view, the most unfavorable case is the one of the forming in variant V2 (trajectory shifted at an angle of $90^{\circ}$ from the previous press point), where this convexity can reach values of up to $0.91 \mathrm{~mm}$. Also a big convexity 


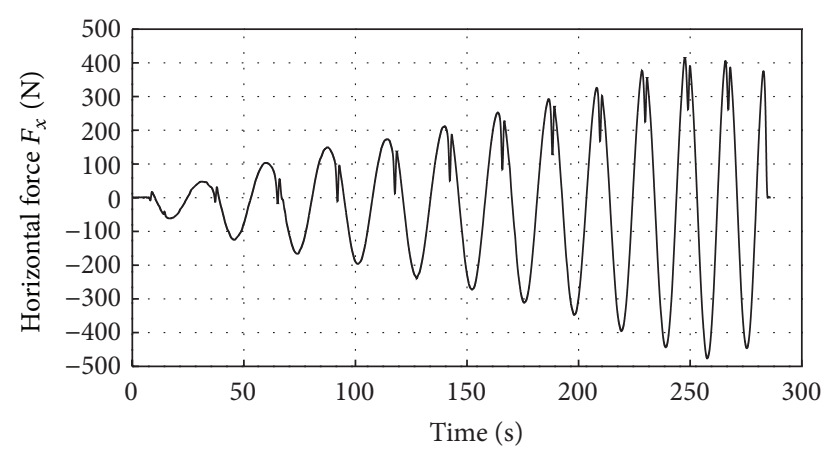

(a)

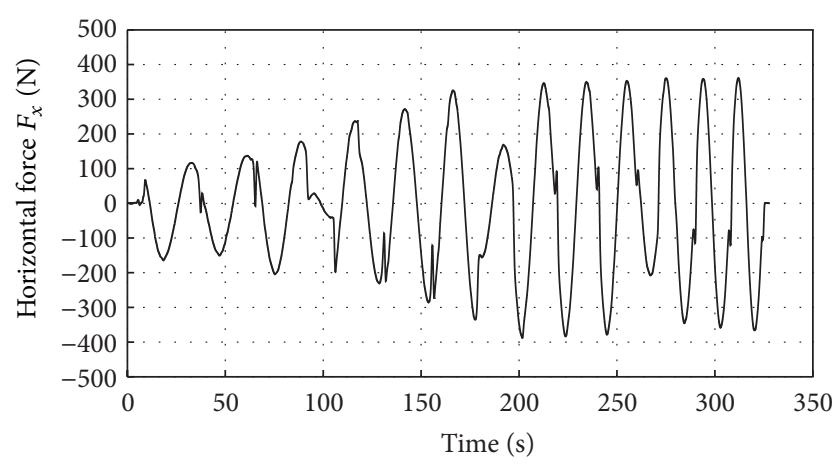

(b)

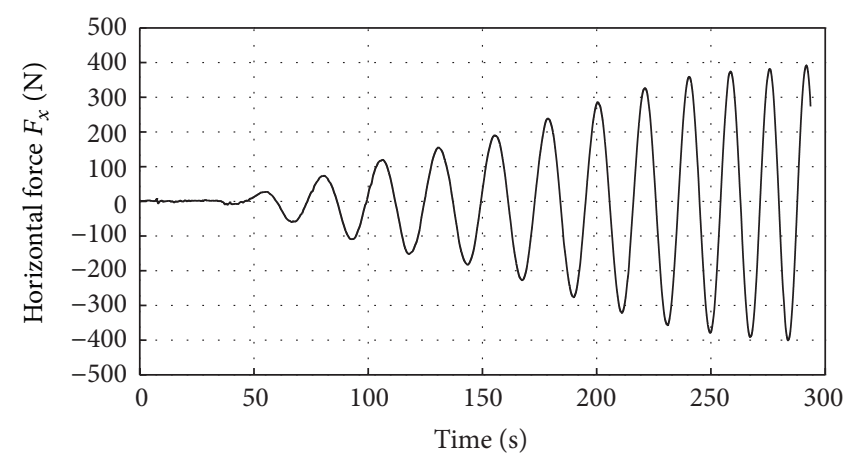

(c)

FIGURE 7: The horizontal force $\left(F_{x}\right)$ variation for the specified three different trajectories.

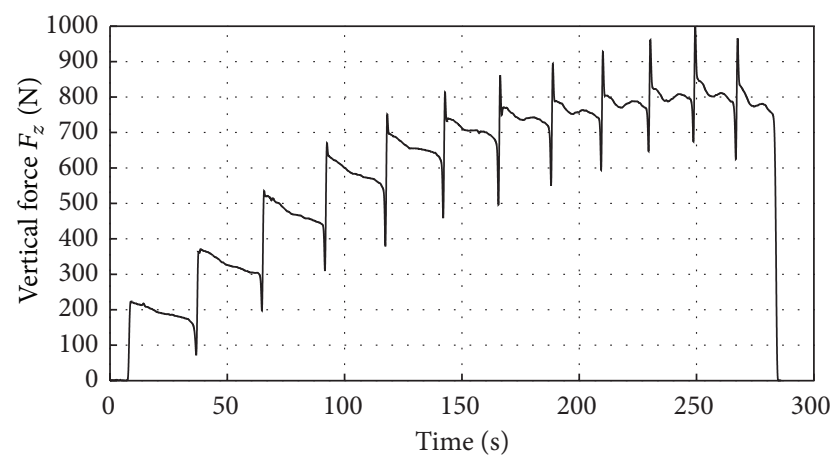

(a)

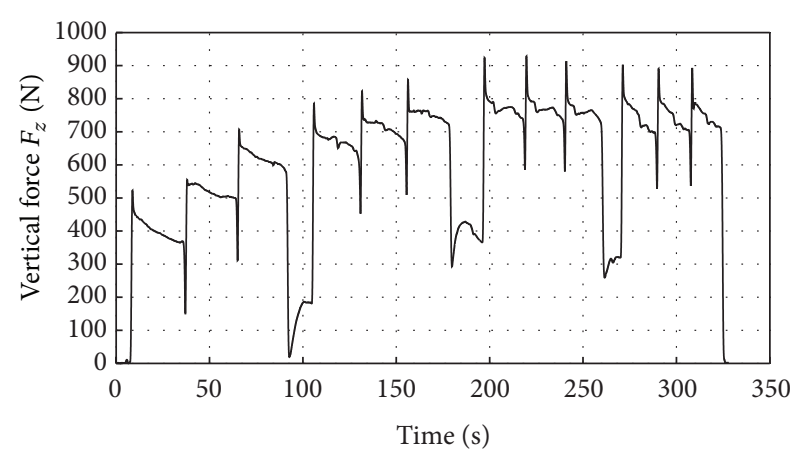

(b)

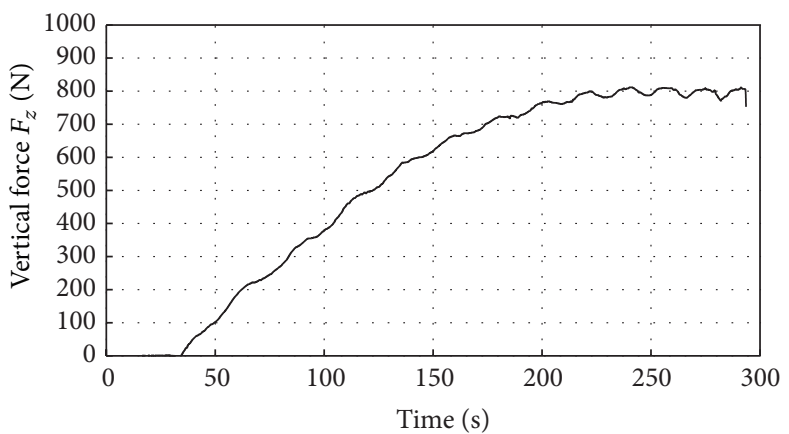

(c)

Figure 8: The vertical force $\left(F_{z}\right)$ variation for the specified three different trajectories. 


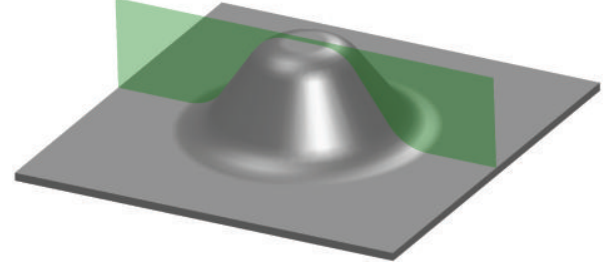

(a)

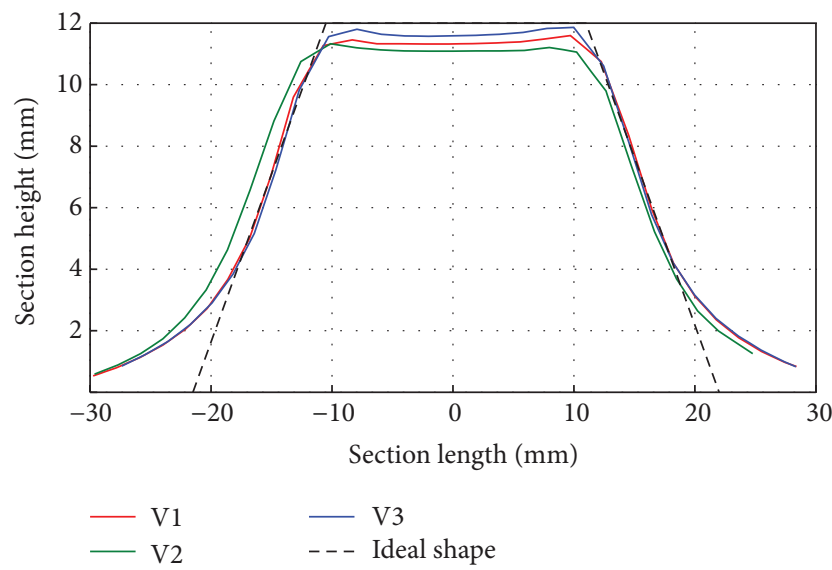

(b)

FIGURE 9: The position of the section plan utilised to measure profiles and comparison of resulting profiles and the ideal shape of the part.

$(0.68 \mathrm{~mm})$ is reached in variant V1 (trajectory which start in the same spot). As can be seen from Figure 9(b), the parts obtained with the variant V3 (spiral trajectory) are the most accurate $(0.41 \mathrm{~mm}$ convexity), although it shows the disadvantages listed above.

This disadvantage can be removed by using a backing plate (TPIF) or minimizing the distance between the forming area and the blankholder. Another disadvantage is the springback. When the punch is removed, the elastic deformations caused by it are removed, and the material tends to recover, and the final form of the piece is slightly smaller than the original. The pillow effect is another disadvantage of SPIF and is present on the small base of the truncated cone parts, causing a slight curvature of the undeformed material in that area.

\section{Conclusions}

The analysis of the strain and force variation of truncated cone-shaped parts indicates that the optimum strain strategy is the one in which the punch follows a spiral path. A reduction of about $18 \%$ in the maximum major strain value occurs in the case of the spiral path compared to the other two strain strategies, and for the minor strain approximately the same maximum value is reached compared to the deflected press strategy; in the case of thickness reduction, a decrease of approximately $13.5 \%$ is obtained compared to the other two strain strategies. Moreover, strains and thickness reduction are evenly distributed for the spiral path compared to the other two strategies.

In the case of force variation, it was noticed that there is not only a decrease in the maximum value for the spiral path strategy of $23 \%$ compared to the press strategy in the same area and of $14 \%$ compared to the strategy of successive presses offset at an angle of $90^{\circ}$ from the previous entry but also a smooth impact-free variation that could lead to the reduction in the dimensional deviations of the parts produced.

In terms of precision of parts obtained through SPIF, the best path is V3, and the resulting profile is the closest to the desired one than in the other two cases.

A future research direction may comprise the study of the strain strategy for parts with different geometries such as truncated pyramid parts, where the strain reaches maximum values due to the geometric structure of the part.

\section{References}

[1] M. Pohlak, J. Majak, and R. Küttner, "Incremental sheet forming process modelling-plimitation analysis," Journal of Achievements in Materials and Manufacturing Engineering, vol. 22, no. 2, pp. 67-70, 2007.

[2] M. B. Silva, M. Skjoedt, P. A. F. Martins, and N. Bay, "Revisiting the fundamentals of single point incremental forming by means of membrane analysis," International Journal of Machine Tools and Manufacture, vol. 48, no. 1, pp. 73-83, 2008.

[3] Z. Liu, Y. Li, and P. A. Meehan, "Vertical wall formation and material flow control for incremental sheet forming by revisiting multistage deformation path strategies," Materials and Manufacturing ProcessesIssue, vol. 25, no. 5, pp. 562-571, 2013.

[4] M. Yamashita, M. Gotoh, and S.-Y. Atsumi, "Numerical simulation of incremental forming of sheet metal," Journal of Materials Processing Technology, vol. 199, no. 1, pp. 163-172, 2008.

[5] J. Li, P. Geng, and J. Shen, "Numerical simulationand experimental investigation of multistage incremental sheet forming," The International Journal of Advanced Manufacturing Technology. In press.

[6] Z. Cui, L. Gao, F. Ren, V. Kiridena, and Z. C. Xia, "Deformation analysis of incremental sheet forming," SAE International Journal of Materials and Manufacturing, vol. 3, no. 1, pp. 732-736, 2010.

[7] M. Bambach, B. Taleb Araghi, and G. Hirt, "Strategies to improve the geometric accuracy in asymmetric single point incremental forming," Production Engineering, vol. 3, no. 2, pp. 145-156, 2009.

[8] M. B. Silva and P. A. F. Martins, "Two-point incremental forming with partial die: theory and experimentation," Journal of Materials Engineering and Performance, vol. 22, no. 4, pp. 1018-1027, 2013.

[9] K. Jackson and J. Allwood, "The mechanics of incremental sheet forming," Journal of Materials Processing Technology, vol. 209, no. 3, pp. 1158-1174, 2009.

[10] F. C. Minutolo, M. Durante, A. Formisano, and A. Langella, "Forces analysis in sheet incremental forming and comparison of experimental and simulation results," in Intelligent Production Machines and Systems, D. T. Pham, E. E. Eldukhri, and A. J. Soroka, Eds., pp. 229-234, Elsevier Science, Oxford, UK, 2006.

[11] J. R. Duflou, B. Callebaut, J. Verbert, and H. De Baerdemaeker, "Improved SPIF performance through dynamic local heating," 
International Journal of Machine Tools and Manufacture, vol. 48, no. 5, pp. 543-549, 2008.

[12] A. Fiorentino, E. Ceretti, A. Attanasio, L. Mazzoni, and C. Giardini, "Analysis of forces, accuracy and formability in positive die sheet incremental forming," International Journal of Material Forming, vol. 2, no. 1, pp. 805-808, 2009.

[13] M. Durante, A. Formisano, A. Langella, and F. M. Capece Minutolo, "The influence of tool rotation on an incremental forming process," Journal of Materials Processing Technology, vol. 209, no. 9, pp. 4621-4626, 2009.

[14] G. Ambrogio, L. Filice, and F. Micari, "A force measuring based strategy for failure prevention in incremental forming," Journal of Materials Processing Technology, vol. 177, no. 1-3, pp. 413-416, 2006.

[15] G. Ambrogio, V. Cozza, L. Filice, and F. Micari, "An analytical model for improving precision in single point incremental forming," Journal of Materials Processing Technology, vol. 191, no. 1-3, pp. 92-95, 2007.

[16] M. Bambach, B. Taleb Araghi, and G. Hirt, "Strategies to improve the geometric accuracy in asymmetric single point incremental forming," Production Engineering, vol. 3, no. 2, pp. 145-156, 2009.

[17] G. Hussain and L. Gao, "A novel method to test the thinning limits of sheet metals in negative incremental forming," International Journal of Machine Tools and Manufacture, vol. 47, no. 3-4, pp. 419-435, 2007.

[18] A. Attanasio, E. Ceretti, and C. Giardini, "Optimization of tool path in two points incremental forming," Journal of Materials Processing Technology, vol. 177, no. 1-3, pp. 409-412, 2006.

[19] M. Rauch, J.-Y. Hascoet, J.-C. Hamann, and Y. Plenel, “Tool path programming optimization for incremental sheet forming applications," CAD Computer Aided Design, vol. 41, no. 12, pp. 877-885, 2009.

[20] Z. Fu, J. Mo, F. Han, and P. Gong, "Tool path correction algorithm for single-point incremental forming of sheet metal," The International Journal of Advanced Manufacturing Technology, vol. 64, no. 9-12, pp. 1239-1248, 2013. 

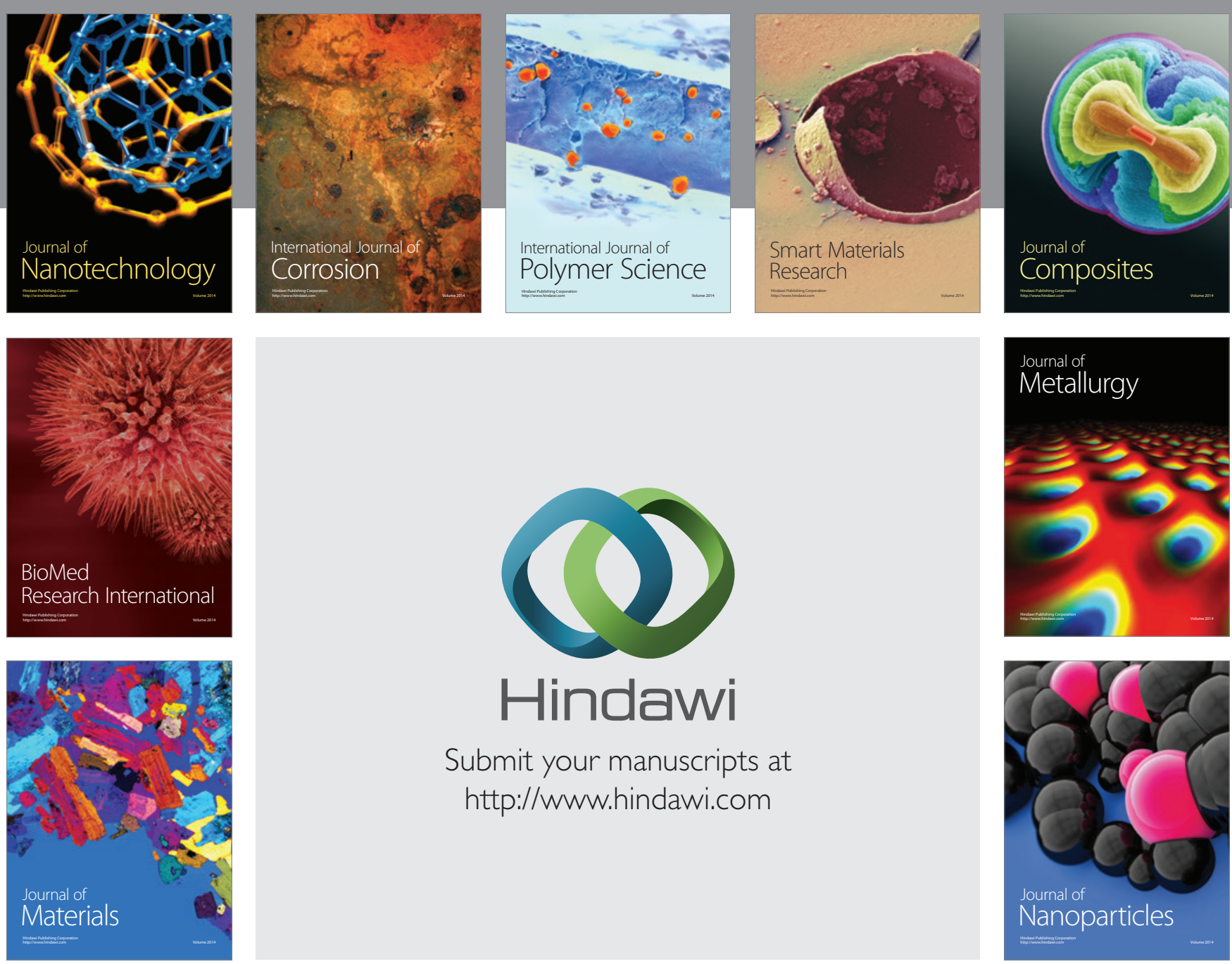

Submit your manuscripts at http://www.hindawi.com
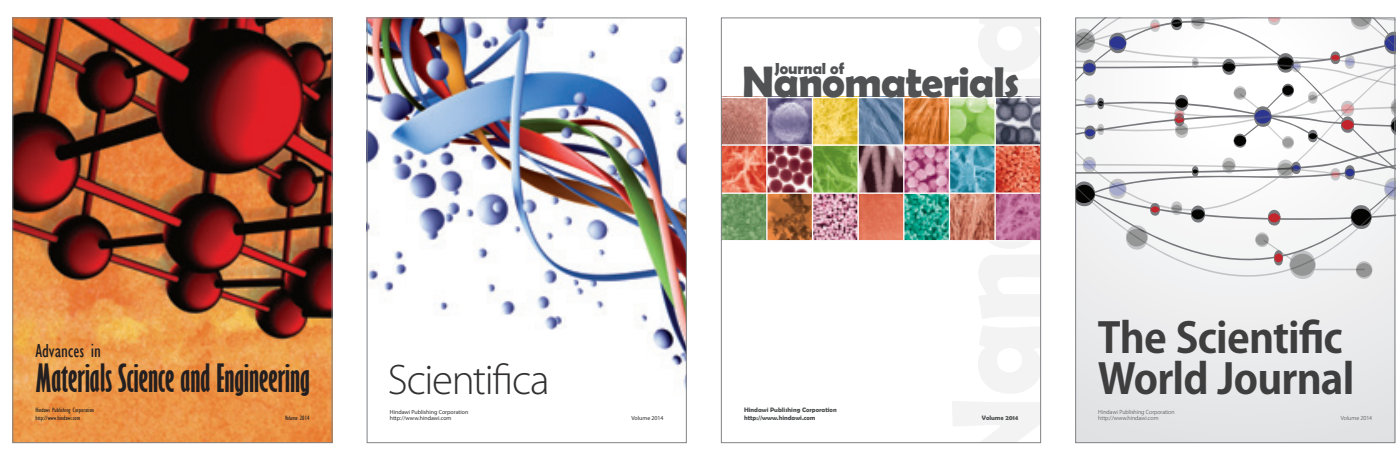

\section{The Scientific World Journal}
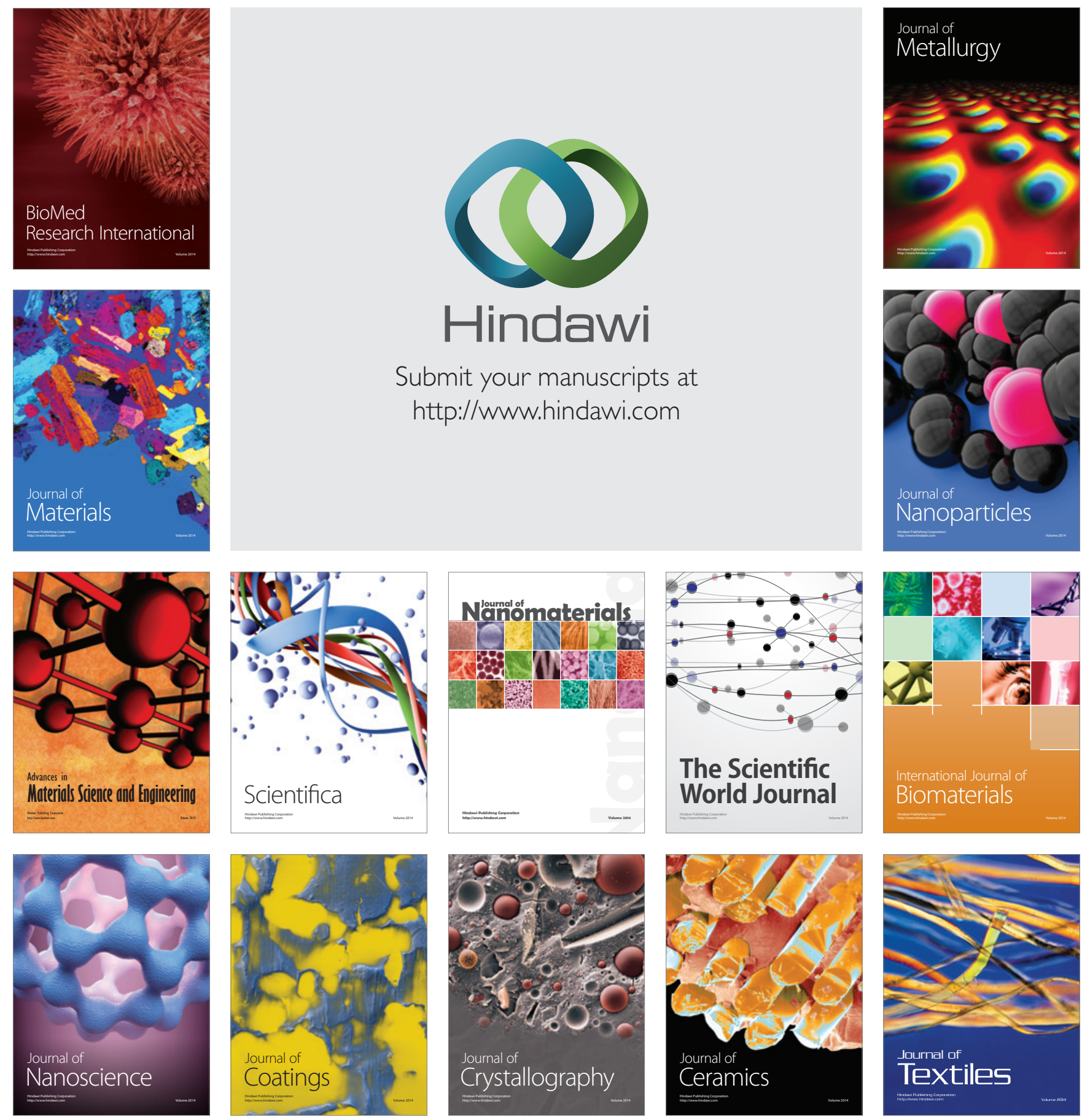